\title{
Differential expression of HSP60 and exercise effects on its levels in Obese Diabetic and Non-Diabetic humans
}

Sina Kavalakatt, Abdelkrim Khadir, Ali Tiss*

Functional Proteomics and Metabolomics Unit, Dasman Diabetes Institute, Kuwait City, Kuwait

\section{Background and Objectives}

Obesity is a growing epidemic, characterized by low grade inflammation and impaired heat shock response(HSR). Physical activity is known to improve overall health by modulating metabolic stress pathways including HSR. Heat shock protein 60 (HSP60), a member of HSR, is a key protein in the crosstalk between cellular stress and inflammation. Interestingly, it can induce both pro-inflammatory and anti-inflammatory markers, however, the status of HSP60 in diabetes and obesity is unclear. We recently reported that HSP60 acting as a chaperonin for proteins in the mitochondrion, was increased in human obese. Its expression levels in obese diabetic are yet to be investigated. In this study, we assessed the expression levels of HSP60 in diabetic and nondiabetic obese (Table 1), along with its correlation with inflammatory and metabolic markers as well as its modulation by physical exercise.

\begin{tabular}{l}
\multicolumn{1}{c|}{ Methods } \\
\hline Study Population, Exercise protocol and anthropometric measurements : Adult male and female subjects consisting of non-diabetic (138) and \\
diabetic (92) obese were recruited in accordance with the Declaration of Helsinki .All eligible subjects were enrolled to a supervised exercise \\
program and anthropometric measurements were taken at baseline and after 3 months of exercise. \\
Blood and tissue sampling: Venous peripheral blood and subcutaneous adipose tissue (SAT) biopsies were obtained at baseline and after 3 \\
months. \\
Blood inflammatory and metabolic markers: Glucose and lipid profiles were measured on Siemens Dimension RXL chemistry analyzer. Insulin \\
and High sensitivity CRP were determined using ELISA kit. Inflammatory and metabolic markers were assessed using Bioplex-20o system. \\
Immunohistochemistry(IHC) and Immunoflourescence (IF) : Formalin fixed paraffin embedded SAT sections were used and IHC quantification \\
was done using Imagescope software. IF was done and analyzed with Zeiss LSM 710 confocal microscope. \\
Quantitative Real time PCR ( qRT-PCR): Total RNA was extracted from frozen SAT using Rneasy Lipid Mini kit and cDNA was synthesized with \\
High capacity CDNA reverse transcription kit. \\
Quantification of circulating proteins by ELISA: Immunoassay EIA kit was used for measuring plasma levels of HSP6o . Levels of plasma anti- \\
HSP6o IgG/A/M were measured using ELISA kit. \\
Statistical Analysis: Statistical analyses were performed using SPSS software (v22.o; SPSS Inc., Chicago, IL, USA). \\
\hline
\end{tabular}

\section{Results}

In this study

HSP6o expression was significantly decreased in SAT of diabetic obese humans compared to non-diabetic obese.(Figure 1) This down regulation was concomitant with increased levels of IL6 and TNFa inflammatory markers in the SAT of diabetic lean and obese. (Figure $2 \& 3$ )

(he lirculating HSP6o and its auto-antibody between these two groups.

The 3-month exercise protocol significantly increased HSP6o expression levels in SAT of diabetic obese along with a decreased inflammation. In non-diabetic obese the same exercise protocol decreased both HSP6o and inflammatory markers TNF and IL6, suggesting a differential exercise effect in the two groups. (Figure 1 \& 2)
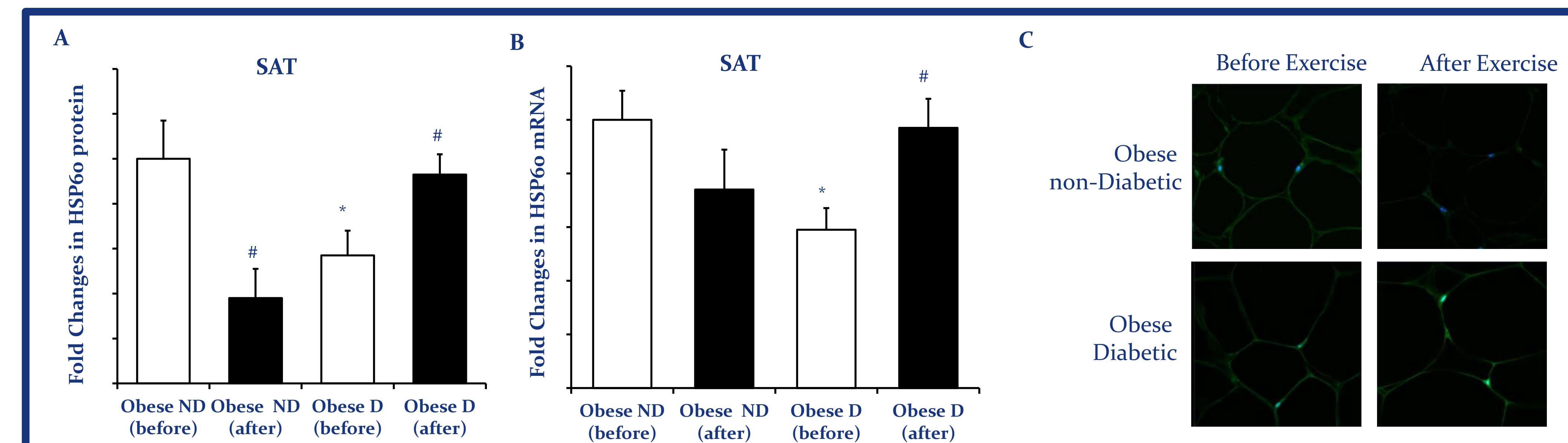

Figure 1: HSP6o expression and modulation by exercise in the subcutaneous adipose tissue (SAT) of obese subjects with diabetes

(A) Immunohistochemical analysis of HSP6o expression in SAT from obese without (ND) and with diabetes (D) before and after
exercise intervention ( $\mathrm{n}=10$ for each group). (B) mRNA levels were measured by quantitative real time PCR using SAT from obese ND

$$
\begin{aligned}
& \text { exerc } \\
& \text { for e } \\
& \text { in SA } \\
& \text { diabe }
\end{aligned}
$$
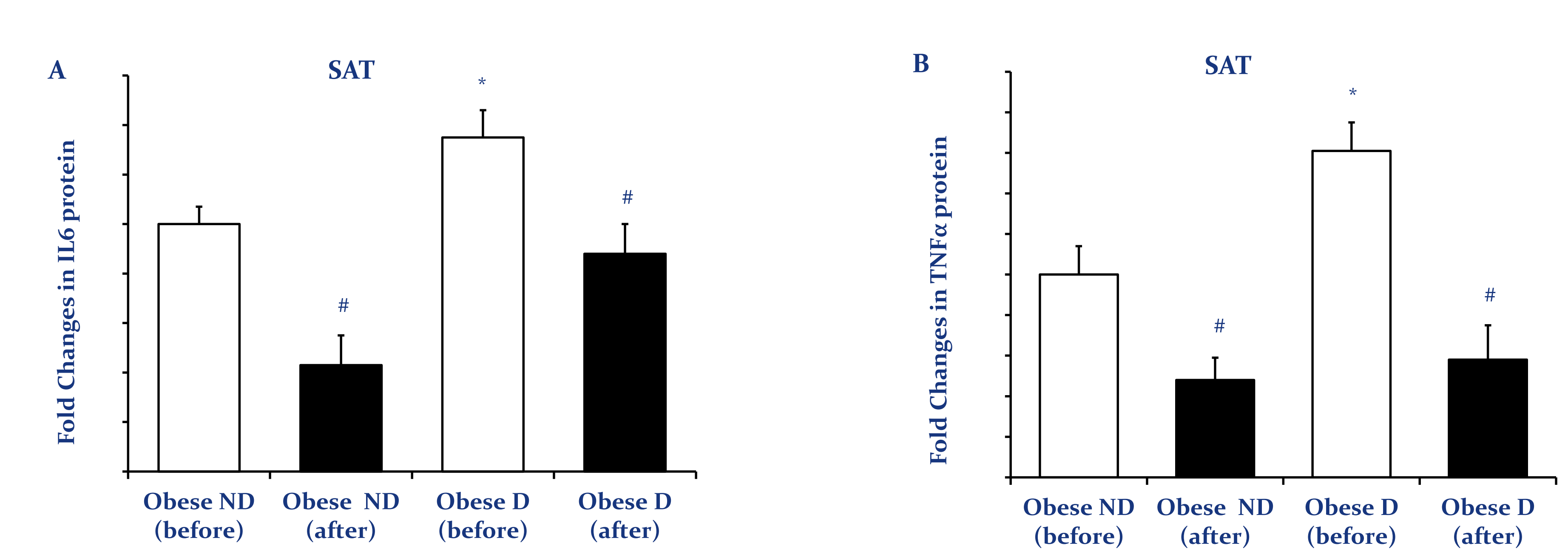$$
\begin{aligned}
& \text { Imr } \\
& \text { mo } \\
& \text { der }
\end{aligned}
$$

\section{References:}

[1]. Tiss A et al. (2014). Lipids in health and disease, 13:106
[2]. Khadir et al. (2016). Am J Physiol Endocrinol Metab, 308, E71-83.

Kuwait Foundation for the Advancement of Sciences (KFAS) for financial support. 